\title{
Los doctorados en los posgrados de Argentina y Brasil
}

$\mathrm{PhD}$ in the national postgraduate education of Argentina and Brazil

\author{
Volumen 21, Número 1 \\ Enero - Abril \\ pp. 1-29
}

\author{
Mónica de la Fare \\ Laura Inés Rovelli
}

\section{Citar este documento según modelo APA}

de la Fare, Mónica., y Rovelli, Laura Inés. (2021). Los doctorados en los posgrados de Argentina y Brasil. Revista Actualidades Investigativas en Educación, 21(1), 1-29. Doi. 10.15517/aie.v21i1.42596 


\title{
Los doctorados en los posgrados de Argentina y Brasil
}

\author{
$\mathrm{PhD}$ in the national postgraduate education of Argentina and Brazil
}

\section{Mónica de la Fare ${ }^{1}$ Laura Inés Rovelli}

Resumen: El artículo indaga las dinámicas recientes de los programas de doctorado en los posgrados en Argentina y Brasil, por lo que se sitúa en el campo temático de los estudios regionales sobre educación superior. El propósito del trabajo consiste en analizar y comparar las principales tendencias de la configuración y desarrollo de los programas doctorales en ambos países en el período 2007-2018. Para ello, se selecciona cuatro dimensiones de análisis: la configuración histórica y regulación del nivel; las características de la oferta y su expansión; la matrícula, graduación e inserción profesional; y el fomento estatal a la formación doctoral. El territorio teórico recupera los estudios sobre educación superior con énfasis en el nivel de posgrado y en la formación de recursos calificados. El abordaje metodológico se inscribe en los estudios comparados en educación y se centra principalmente, en datos cuantitativos. Los resultados sostienen una tendencia convergente en las dinámicas de expansión reciente de los programas y matrículas doctorales en ambos países, aunque se observan contrastes significativos en el plano de la regulación del nivel, la modalidad de la oferta y su interacción con otros subsistemas, cuestión ligada estrechamente con la configuración histórica del nivel y sus políticas.

Palabras clave: grado de doctor, educación de posgrado, Argentina, Brasil.

Abstract: The article investigates the recent dynamics of postgraduate doctoral programs in Argentina and Brazil, and thus it is situated in the thematic field of regional studies on higher education. The purpose of the work is to analyze and compare the main trends in the configuration and development of doctoral programs in both countries in the period 2007-2018. For this, four dimensions of analysis are selected: the historical configuration and regulation of the level; the characteristics of the offer and its expansion; enrollment, graduation and professional insertion; and state promotion of doctoral training. The theoretical territory includes studies on higher education, with an emphasis on the postgraduate level and the training of qualified resources. The methodological approach is part of comparative studies in education and focuses mainly on quantitative data. The results support a convergent trend in the dynamics of recent expansion of doctoral programs and enrollments in both countries, although significant contrasts are observed in the level of regulation of the level, the modality of the offer and its interaction with other subsystems, a related issue closely with the historical configuration of the level and its policies.

Keywords: doctoral degrees, postgraduate education, Argentina, Brazil.

\footnotetext{
${ }^{1}$ Docente investigadora de la Pontifícia Universidade Católica do Rio Grande do Sul (PUCRS), Programa de Posgrado en Educación, Porto Alegre, Brasil. Dirección electrónica: monica.fare@pucrs.br Orcid: https://orcid.org/0000-0002-2660-4043

2 Investigadora del Consejo Nacional de Investigaciones Científicas y Técnicas (CONICET), y del Instituto de Investigaciones en Humanidades y Ciencias Sociales (IdIHCS), Universidad Nacional de la Plata (UNLP), Buenos Aires, Argentina. Dirección electrónica: laurarovelli@gmail.com Orcid: https://orcid.org/0000-0001-7059-149X
}

Artículo recibido: 30 de junio, 2020

Enviado a corrección: 9 de setiembre, 2020

Aprobado: 9 de diciembre, 2020 


\section{Introducción}

El espacio social del posgrado en Argentina y Brasil se encuentra atravesado desde sus inicios por la apropiación de modelos extranjeros. La institucionalización de los primeros programas se nutre de la captación de personas con título de doctorado en otros países, quienes contribuyen a conforman un cuerpo docente acreditado y a fortalecer el intercambio y la cooperación internacional en sus ofertas. No obstante, la trayectoria histórica de los doctorados y las tradiciones universitarias que los moldean, así como también la orientación por parte del Estado y las políticas de reforma del nivel imprimen singularidades propias a su configuración en cada espacio nacional, cuestión que enriquece la comparación y, a la vez, fundamenta la elección de los dos casos priorizados.

Un informe de la Organización para la Cooperación y el Desarrollo Económico (OCDE) destaca, entre 1998 y 2014, un aumento del número de personas con título de doctorado en el mundo, cifra que en los países con economías consideradas emergentes asciende al $24 \%$ del total de la población titulada en ese nivel (OCDE, 2016). En ese escenario internacional, en los países seleccionados para este estudio, se evidencia un marcado incremento de las carreras de doctorado, las matrículas y la graduación. Sin embargo, recientemente, la dirección política por parte de los gobiernos nacionales muestra, en el caso de Brasil, un panorama complejo y de incertidumbre ante la retracción de ciertos programas y la desinversión en el sector universitario y científico, mientras que en Argentina, luego de un período regresivo en ese ámbito y su financiamiento, entre diciembre de 2015 y 2019, vuelve a tener continuidad y se fortalece, a partir de 2020, la directriz de formación de recursos humanos calificados y la alternativa de inserción en la carrera en ciencia y tecnología ${ }^{3}$.

Este artículo se suma a una producción acumulada sobre el tema del posgrado, que aumenta en los últimos años, pero que aún puede ser caracterizada como incipiente y con tendencia a la realización de estudios nacionales. Ambos países adquieren una importancia estratégica en el escenario regional latinoamericano y mantienen una fuerte influencia e intercambios mutuos, cuestiones que justifican la realización de esta investigación. A su vez, son aún más escasos los trabajos que focalizan en el nivel de doctorado, característica que alentó la indagación, en clave comparativa, de las características que distinguen esos

\footnotetext{
${ }^{3}$ En el caso de Brasil, nos referimos al gobierno del presidente Jair Messias Bolsonaro, quien asume en su cargo en enero de 2019. Mientras que, en Argentina, el primer período mencionado corresponde al gobierno de la Alianza Cambiemos, liderada por Mauricio Macri. Y el segundo ciclo gubernamental, iniciado en diciembre de 2019 y aún en curso, es ejercido por el Frente de Todos, conducido por el actual presidente Alberto Fernández.
} 
programas. En ese marco, el objetivo principal del trabajo busca analizar las dinámicas recientes de expansión y consolidación de los programas de doctorado en Argentina y Brasil. Para ello, se priorizan y comparan cuatro dimensiones: la configuración histórica y los modos de regulación del nivel; las características de la oferta y su expansión; la matrícula, la graduación e inserción; y el fomento estatal a la formación doctoral.

\section{Referentes teóricos}

Al analizar la agenda de producción de conocimiento sobre el posgrado en los dos países, es posible identificar su acompasamiento con el ciclo de la política de educación superior. Es decir, a una primera tematización sobre las dinámicas de expansión en los años 90 del siglo pasado le sigue la reflexión acerca de la orientación de la formación en la primera década de los años 2000 para dar lugar, en los últimos 10 años, a la problematización en torno a la inserción laboral y profesional de las personas doctoradas. Aunque el escenario de la producción puede ser extendido a varios países de la región, el estado del arte que se presenta en este apartado se limita al universo de los países priorizados.

En Brasil, la producción sobre este tema está integrada por un conjunto de trabajos provenientes de distintos ámbitos. Se verifica la existencia de publicaciones del campo de la investigación académica, así como también planes e informes nacionales que incluyen el análisis de la política de ese nivel académico. Estos documentos han sido elaborados por iniciativa del principal organismo de gobierno de la educación encargado de la gestión nacional del posgrado, la Coordenação de Perfeccionamiento de personal de la Educación (CAPES) del Ministerio de Educación, que también edita una revista especializada (Revista Brasileira da Pos-graduação).

Un trabajo frecuentemente citado (Balbachevsky, 2005) analiza el posgrado en ese país a partir de una reconstrucción de su historia, en diálogo con una producción pionera sobre la ciencia brasileña (Schwartzman, 1991). Posteriormente, los estudios sobre el sistema de posgrado en ese país reconocen, para el período 1998-2011, un proceso de expansión y fortalecimiento de los programas. Entre otras dimensiones toman en consideración: los estándares y el control ejercido por el sistema de evaluación, la inversión continua de recursos en becas de formación e intercambio internacional, el crecimiento de matrículas y el incremento de los egresados, así como la cantidad de docentes incorporados a ese nivel de la educación superior y la distribución regional que ha experimentado cambios pero que aún presenta desequilibrios (Ciriani, Campario y Silva, 2015; Moritz, Moritz, Pereira y Maccani, 
2013). Junto a esas producciones referidas al conjunto del posgrado se identifican abundantes trabajos referidos a delimitaciones establecidas para áreas de conocimiento específicas (Hortale, Moreira, Bochner, y Leal, 2014; Kuenzer y Moraes, 2005; Mendes, Werlang y Santos, 2017; entre otros).

También, se reconocen producciones dedicadas a analizar una de las características novedosas de este sistema, como es la emergencia a mediados de los años 2000 y posteriormente, de la expansión de carreras de maestrías profesionales en un posgrado estructurado sobre la base de una tradición de carreras académicas y la creación de carreras de doctorado profesional, que ya comienzan a tener personas graduadas (Castro, 2005; Negret, 2008). En esa dirección, comienzan a ser publicados trabajos que abordan el tema de la oferta de doctorados profesionales y en parte recuperan la discusión presente en las asociaciones profesionales (Calderon et al, 2019).

En Argentina, los abordajes sobre la temática se desarrollan con mayor sistematicidad en los años noventa, ligados a la institucionalización del campo de la universidad como objeto de estudio (Barsky, 1995; García de Fanelli, 1996; Krotsch, 1996). En los últimos veinte años surgen investigaciones que analizan la trayectoria histórica y las dinámicas de expansión del posgrado en relación con las políticas universitarias y científicas del país. Un estudio integral realizado en el campo universitario argentino, entre 1995 y 2009, señala que, si bien, el aumento de las carreras posgrado en esos años incluye a todos los campos del conocimiento es exponencial en aquellas de orientación profesionalista (en especial, en las carreras de especialización y maestría) y de manera más acentuada, en el ámbito de las universidades de gestión estatal (de la Fare y Lenz, 2012). A su vez, la investigación afirma que las restricciones presupuestarias desplegadas por las políticas universitarias de los años noventa alientan estrategias de mercado en el marco de tendencias mundiales hacia la mercantilización y la privatización de la educación superior (de la Fare y Lenz, 2012). A partir de esa expansión, un grupo de producciones más recientes profundizan distintas dimensiones vinculadas a la configuración del espacio del posgrado. Algunos trabajos problematizan, en clave comparada y en el plano regional, la evaluación y acreditación de las carreras (Araujo, Balduzzi, Corrado y Walker, 2016), y la situación actual de carreras, estudiantes y egresados en Argentina, Brasil y Paraguay (García, 2016), entre otras temáticas.

Otro conjunto de trabajos identifica un segundo período de expansión del posgrado, con posterioridad a la crisis socioeconómica y política de 2000- 2002 en el país, entre los años 
2003 y $2015^{4}$, en el que aún cuando perviven algunas de las tendencias de los años noventa, se despliega una orientación más consolidada de las políticas de posgrado, y en particular, de los doctorados. Como resultado de una mayor inversión pública en ciencia y tecnología se produce una ampliación significativa de las becas de formación doctoral por parte del principal organismo estatal de financiamiento, el Consejo Nacional de Investigaciones Científicas y Técnicas (CONICET), cuestión que incide en el sobredimensionamiento de los programas de doctorado ofertados principalmente por las universidades nacionales (Gallardo, 2015; Jeppesen, Golberg, Szpeiner y Rodríguez Gauna, 2015; Unzué, 2011). De allí que, recién en este período, el nivel de Doctorado comience a ser objetivado de manera privilegiada y más extendida en los estudios sobre posgrado con una menor tradición en el nivel, como es el caso de las ciencias humanas y sociales, y en particular en el área de Educación.

En ese marco, diversas investigaciones problematizan la inserción profesional de los posgraduados ante la formación predominantemente académica de la formación y el estrangulamiento laboral, en particular de los nuevos doctores, que dificulta su ingreso a los ámbitos científicos y universitarios existentes (Emiliozzi, 2013; Unzué, 2013; 2015). A lo anterior se suman nuevas indagaciones que comienzan a reflexionar sobre la orientación de la formación doctoral y los instrumentos de política direccionados a temas estratégicos definidos en el país (Jeppesen, Bentura, Golberg y Fernández López, 2018; Rovelli, 2017). Cabe destacar que la mayor parte de los trabajos coinciden en afirmar la brecha existente entre el aumento de personas graduadas en el nivel de doctorado y la disponibilidad de posiciones existentes en la academia y las instituciones científicas (Unzué, 2013).

A modo de balance, podemos considerar que tanto en Brasil como en Argentina se ha desarrollado en las últimas dos décadas una incipiente producción de estudios nacionales dedicados al análisis del posgrado, y en menor medida, sobre sus implicancias y dinámicas recientes en el nivel doctoral.

\footnotetext{
${ }^{4}$ En Argentina, este segundo período de expansión del posgrado ocurre en paralelo a mayor inversión pública en ciencia y tecnología y educación superior, junto con la expansión de becas para la formación doctoral durante los llamados gobiernos progresistas de Néstor Kirchner (2003-2007) y los dos mandatos posteriores de Cristina Fernández de Kirchner (2007-2015).
} 


\section{Metodología}

\subsection{Enfoque}

El enfoque metodológico se inscribe en los estudios comparados en educación, los cuales cuentan con una prolífica literatura que incluye polémicas en relación con su definición, así como un amplio conjunto de estudios alineados con ese abordaje de investigación. Algunas perspectivas la definen como un espacio que nuclea producciones que optan por el contexto comparado para abordar temas educativos y que para eso recurren a abordajes y herramientas provenientes de diferentes campos de conocimiento (Bray, Adamson y Mason, 2010). Es esa la concepción que permea este trabajo, en el que se enfatiza la relevancia de la historicidad de los fenómenos para comprender la singularidad de los procesos nacionales, sin dejar de considerar la influencia de tendencias internacionales (Piovani y Krawczyk, 2017).

\subsection{Unidad de análisis, población de estudio o participantes}

Los programas de doctorado acreditados por los sistemas de evaluación de ambos países, la Comisión Nacional de Evaluación y Acreditación Universitaria (CONEAU) en Argentina y la Coordenação de Aperfeiçoamento de Pessoal de Nível Superior (CAPES) en Brasil, son las unidades de análisis. Para ello, se ha privilegiado el estudio intensivo de esos dos casos nacionales y las siguientes dimensiones cuantitativas: el número de carreras, la categorización de los programas, la distribución regional, las matrículas, las becas de fomento a la formación doctoral, los graduados y la inserción en las universidades.

\subsection{Técnicas de recolección de datos}

En una primera etapa se realiza una revisión de la historia de los posgrados en ambos países a partir del análisis de datos cualitativos provenientes de fuentes primarias, principalmente documentos oficiales, y de fuentes secundarias. Se busca comprender las convergencias y diferencias, enfocadas en el nivel de doctorado, contextualizadas en las universidades en las que se insertan. Posteriormente, se recurre al análisis de datos cuantitativos de las estadísticas oficiales nacionales del periodo 2007-2017 y según las fuentes de información, en algunos casos, se consideran los años 2008-2018. Con ello, se pretende construir una lectura interpretativa atravesada por la inserción profesional de las autoras en programas doctorales de ambos países.

El relevamiento y análisis de la información estadística disponible evidencia una diferencia importante entre los casos nacionales. En Brasil, se dispone de un sistema 
estadístico público georreferenciado organizado por la CAPES, que ofrece un amplio y actualizado conjunto de informaciones sobre ese nivel, en concordancia con la política de datos abiertos del Poder Ejecutivo Nacional, fijada para ese organismo por el Decreto $N^{\circ} 8777$, del 11 de mayo de 2016. En Argentina, los datos oficiales sobre matrícula, personas egresadas y oferta de carreras del sistema de educación superior y, en particular, del nivel de posgrado, se encuentran dispersos entre distintos organismos gubernamentales. Por su parte, la oferta de becas de formación doctoral es sistematizada por uno de los organismos principales de financiamiento y promoción científica, el Consejo Nacional de Investigaciones Científicas y Técnicas (Conicet). Dicha fragmentación y la ausencia de un sistema nacional de información estadística complejiza el análisis y exige un trabajo minucioso para elaborar la comparación, a la vez que hace necesario adaptar los términos y períodos ante la información existente.

Ese proceso analítico consistió en la identificación y selección de las fuentes de informaciones estadísticas oficiales por parte de la CONEAU y la Secretaría de Políticas Universitarias (SPU) para los datos sobre carreras, matrícula y egresos, y el CONICET, para becas de formación doctoral en Argentina y exclusivamente el Sistema GEOCAPES, en Brasil. Paralelamente, el aporte de las metodologías empleadas para la cuantificación y de las distintas características de la evaluación en cada caso nacional nutrieron el estudio a fin de avanzar posteriormente en la selección y recopilación de datos comparables, tomando en consideración las variables y series estadísticas disponibles. Esto llevó a identificar el período 2008-2018 para analizar la expansión de carreras y el de 2007-2017 para contrastar la evolución de matrículas y graduados, así como la distribución regional de estos últimos. En el caso de las becas de fomento a la formación doctoral se optó por priorizar al CONICET en Argentina y a la CAPES en Brasil, en virtud del predominio cuantitativo de sus respectivas ofertas respecto del resto de las agencias y/o instituciones financiadoras existentes. Asimismo, se decidió extender el análisis al período 2007-2018 en función de la disponibilidad de datos más recientes. A su vez, se seleccionaron las dimensiones de análisis ya presentadas y se tomó la decisión de trabajar con el universo de datos sobre doctorados acreditados, considerando que en Brasil la acreditación siempre implica categorización, mientras que en Argentina la acreditación no incluye, en todos los casos, categorización.

\subsection{Procesamiento de datos}

Los datos relevados han sido sistematizados en una hoja de cálculo, lo que permite visualizar las distintas dimensiones de análisis y su categorización, así como también ejercitar 
el análisis comparativo mediante la identificación de las principales diferencias y similitudes entre los casos nacionales seleccionados. Posteriormente, se presenta esa información en el formato de ocho tablas. Finalmente, para la interpretación de los datos se ha desplegado un diálogo constante entre la teoría, la configuración histórica y situada de los casos y la base empírica relevada.

\section{Resultados}

\subsection{Configuración histórica y regulación}

Las primeras iniciativas de estudios de posgrado emergen de forma similar en ambos países, a mediados del siglo pasado y vinculadas a la formación de núcleos institucionales reducidos en algunas áreas de conocimiento. Se inspiran en modelos extranjeros, basados en relaciones tutoriales de docencia e investigación entre el profesorado y las personas en formación. No obstante, alcanzan poca repercusión en el conjunto de las universidades. La institucionalización más amplia de los doctorados recién se produce en los años 70 , en Brasil y en los 90, en Argentina y da lugar a hibridaciones y configuraciones propias.

En el primero de esos países se identifican dos antecedentes principales de creación del posgrado: el Parecer del Consejo Nacional de Educación nº 977 de 1965 (Conselho Federal de Educação da República Federativa do Brasil, 1965), presentado por iniciativa del intelectual brasileño Newton Lins Buarque Sucupira (1920-2007). Más conocido como Parecer Sucupira reglamenta inicialmente el posgrado, explícitamente basado en el modelo norteamericano ${ }^{5} \mathrm{y}$ diferencia dos categorías de carreras: lato sensu, especializaciones y stricto sensu, maestrías y doctorados. En segundo lugar, la reforma universitaria de 1968 adopta la articulación de las carreras en un sistema departamental, como resultado del abandono del modelo de cátedras. A dichos departamentos se articulan las carreras de posgrado stricto sensu, con cierto grado de autonomía (Balbachevsky, 2005). Como destaca esta autora, la formación doctoral en el sistema de organización por cátedras se limitaba a algunos nichos pero sin alcance nacional, principalmente vinculados a las Ciencias Exactas y Naturales. Por el contrario, la organización departamental dinamizó la expansión de esas carreras en el conjunto de las universidades (Balbachevsky, 2005).

\footnotetext{
5 "El posgrado - el nombre y el sistema - tiene su origen próximo en la propia estructura de la universidad norteamericana..." (Capes, 1965: 2, traducción propia).
} 
Si bien, las principales agencias de fomento de la investigación científica y promoción de la formación de recursos humanos altamente calificados, el Conselho Nacional de Desenvolvimento Científico e Tecnológico (CNPq) del Ministerio de Ciencia, Tecnología, Innovaciones y Comunicaciones y la CAPES, del Ministerio de Educación habían sido creadas en la década de 1950, el salto de calidad del posgrado se atribuye a las políticas de Ciencia y Tecnología de la década de 1970. Éstas se orientaron a la vinculación entre el desarrollo científico y económico, en el marco de una dictadura militar de orientación nacionalista (Schwartzman, 1991). También fue a mediados de esa década que se institucionalizó el sistema de evaluación por pares (Balbachevsky, 2005), que continua hasta la actualidad, aunque habiendo experimentado varias modificaciones.

Existe consenso entre quienes investigan la temática (Balbachevsy, 2005; Ciriani et al., 2015; Moritz et al., 2013; entre otros) en considerar que el sistema de posgrado stricto sensu de ese país es exitoso y que su desarrollo se liga a políticas gubernamentales de larga duración, en las que se destaca el sistema nacional de becas, financiado por la CAPES y el CNPq. A lo anterior se suma el fomento, aunque en una escala mucho menor, de las Fundaciones de Investigación de los gobiernos estaduales.

La prioridad del posgrado, aunque con diferentes intensidades, se mantiene en las últimas décadas y se intensifica en los años 2000, en el marco de un proceso general de expansión del sistema universitario hasta la novedad producida por el gobierno nacional actual que a través de un conjunto de declaraciones inéditas referidas a la educación superior, avanza en una política de desprestigio y desinversión en el sistema universitario y científico tecnológico ${ }^{6}$. Entre otras medidas, pretende intervenir en el gobierno de las universidades a través de la designación de rectores temporarios por parte del Ministerio de Educación, desconociendo todos los principios democráticos que rigen esas instituciones; propone la eliminación del CNPq y su unificación con la CAPES, cuestión que tendría serias repercusiones en la ciencia y la formación científica brasileñas. Asimismo, ha establecido áreas estratégicas que excluyen en las convocatorias de fomento numerosos campos de conocimiento, especialmente los de las Ciencias Humanas y Sociales, así como también redefine la distribución de becas de formación de posgrado, con criterios confusos y poco explicitados.

6 Nos referimos al gobierno de Jair Massias Bolsonaro, quien asumió la presidencia en Brasil el 1ro de enero de 2019. 
En cuanto a la regulación del sistema de posgrado, en Brasil, la CAPES a partir de la creación del sistema de evaluación por pares en la década de 1970, adquiere capacidades para regular ese espacio e incentivarlo a través del financiamiento vinculado a la evaluación de carreras stricto sensu, a los que se les asigna una categoría numérica (en el caso de los doctorados, desde 7 que es la máxima a 3 que es la mínima). Dicho sistema se estructura a partir de tres dimensiones: evaluación por pares; naturaleza meritocrática que lleva a una clasificación de las carreras en los distintos campos disciplinares; asociación de reconocimiento y fomento como definición de políticas y criterios para la distribución del financiamiento (CAPES, 2010).

Entre otras acciones, ese organismo motoriza y organiza la realización de Planes Nacionales de Posgrado (PNPG), iniciados en la década de 1970. El actual es el sexto, corresponde al periodo 2011-2020 (CAPES, 2010) y es el primero de esos instrumentos articulado al último Plan Nacional de Educación 2014-2024 (Ley n. 13.005/2014), que incorpora como una de sus metas alcanzar la formación anual de 60.000 magísteres y de 25.000 doctores. En la fase de conclusión, el PNPG es evaluado por una comisión de especialistas (CAPES, 2018b), quienes han sugerido un conjunto de modificaciones que actualmente orientan un proceso de redefinición de criterios e instrumentos de evaluación, liderado por la CAPES. Esos cambios en la evaluación buscan implantar un modelo multidimensional, ya aprobado por el Consejo Superior de esa agencia, basado en la evaluación numérica de cada una de las siguientes áreas: formación; investigación; innovación y transferencia de conocimiento; impacto en la sociedad e internacionalización (CAPES, 2018b). Así, los Programas dejarán de tener una nota clasificatoria única, como es actualmente y pasarán a tener cinco, correspondientes a cada una de las dimensiones mencionadas.

Otra novedad es la sustitución del sistema brasileño de evaluación y clasificación de revistas (denominado QUALIS), por el uso de indicadores internacionales para la evaluación de las publicaciones, que serán definidos por las áreas de conocimiento, decisión que genera amplias discusiones. Sumado a lo anterior, los cambios en curso introducen la disminución del número de áreas de evaluación, que actualmente son 49, a través de la unificación de varias de ellas.

En Argentina, a comienzos del siglo XX y en el marco de la institucionalización de la investigación científica en las universidades surgen los primeros doctorados. Así, en el contexto de proyectos institucionales innovadores, como el de la Universidad Nacional de La Plata, se crean los doctorados en Ciencias Naturales, Física, Ciencias Exactas, Ciencias 
Jurídicas y Sociales, Filosofía y Letras, Agronomía y Veterinaria y Ciencias de la Educación. Por otra parte, se fundan los doctorados en Bioquímica y Farmacia en la Universidad de Buenos Aires, en la Universidad Nacional de Rosario y en la Nacional de Tucumán. No obstante, a partir de la década de 1930 y la siguiente, la inestabilidad política en el país incide en la vida universitaria, al generar la cesantía de muchos miembros del profesorado y un fuerte retroceso en la actividad científica, cuestión que frena el crecimiento de los primeros doctorados y de manera más amplia, debilita el surgimiento del nivel de posgrado (Barsky, 1995).

A mediados del siglo pasado se desarrolla un proceso de modernización universitaria y científica, que profesionaliza en ciertos ámbitos institucionales la actividad académica mediante la sustanciación de concursos, el incremento de las dedicaciones exclusivas y la promoción de la organización departamental, en detrimento de la estructura más tradicional y arraigada de cátedras, que aún permanece en las universidades más tradicionales. En ese escenario, proliferan las carreras de posgrado limitadas a algunos nichos de conocimiento, en particular en el área de las Ciencias Sociales y las Médicas. De esta manera, la configuración del posgrado en el país importa distintos modelos extranjeros propios de la tradiciones europeas (con énfasis en la francesa) y norteamericana, los que se superponen como capas o legados de distintos momentos de modernización, y conforman un modelo más bien híbrido (de la Fare y Lenz, 2012). A su vez, en 1958, a partir de la creación del CONICET, inspirado en el modelo francés, el Centre National de la Recherche Scientifique (CNRS), se implementa un diseño de carrera científica y tecnológica donde el organismo estatal es el proveedor de los cargos y evalúa el acceso, permanencia y promoción a la carrera científica sin necesidad de vinculación con un cargo docente en alguna universidad (Lovisolo, 2000).

En los años oscuros de la última dictadura cívico-militar, los proyectos de modernización quedan inconclusos, a la vez que el régimen autoritario avanza en desmantelar la investigación en las universidades públicas, a partir del cierre de carreras, persecución, desaparición, exilio de docentes e intervención en las instituciones. En paralelo, tiene lugar una transferencia de recursos hacia el CONICET, observable en la asignación creciente de fondos al organismo y en la multiplicación de institutos, centros y programas bajo su jurisdicción, cuestión que produce un quiebre importante en la relación entre el sector universitario y el científico (Bekerman, 2019).

A partir de la transición democrática de la década de 1980, se recupera lentamente la vida institucional de las universidades, a la vez que se implementan las primeras elecciones 
de autoridades, en el marco de la tradición del gobierno tripartito. A su vez, el CONICET reconfigura la oferta de becas de formación y de subsidios para el desarrollo de la investigación en convocatorias públicas (Hurtado, 2015). En ese escenario, surge una primera iniciativa de diseño de una política de posgrado, a través de la creación el Sistema Interuniversitario de Cuarto Nivel (SICUN) ${ }^{7}$. No obstante, este antecedente es obturado y sus objetivos principales frustrados ante distintos condicionamientos organizativos, el conflicto en torno a diversos proyectos institucionales en pugna y en definitiva, la ausencia de una política universitaria orientadora para el conjunto del sistema, cuestión que ante el avance de la modernización neoliberal de los años noventa favorece la expansión exponencial de carreras de posgrado.

A partir de la década de 1990, en el marco de una nueva relación entre el Estado y las universidades orientada - entre otros ejes- a introducir la evaluación y el aseguramiento de la calidad como principales directrices de la política, la sanción en 1995 de la Ley $n^{\circ} 24.521$ de Educación Superior (LES), establece un marco regulatorio común para la apertura, funcionamiento y acreditación del posgrado. A su vez, crea un organismo descentralizado, la Comisión Nacional de Evaluación y Acreditación Universitaria (CONEAU), encargada de evaluar y acreditar periódicamente las carreras de especialización, maestría y doctorado en el ámbito de las instituciones universitarias, los centros de investigación e instituciones de formación profesional superior, de acuerdo a los criterios y parámetros establecidos por el Ministerio de Educación y el Consejo de Universidades en el ámbito nacional (Lvovich, 2010). En ese marco, la oferta de carreras de posgrado se expande de manera exponencial a partir de esa década, primero a través de la creación de especializaciones y maestrías, y posteriormente de los doctorados.

El posgrado argentino es regulado por la CONEAU a partir de dispositivos de aseguramiento de la calidad desplegados en la evaluación y acreditación periódica, que establece categorías de $A$ (máxima) a C (mínima) sólo cuando son solicitadas por los programas. No obstante, hasta la actualidad, su expansión no está ligada a planes nacionales de ciencia y tecnología o a metas específicas de la política universitaria, lo que repercute en su escasa coordinación y planificación (Lvovich, 2010) y en la existencia de mayores márgenes de autonomía por parte de las universidades en la implementación de políticas (Unzué, 2013). A esto se suman los escasos subsidios e incentivos por parte del Estado para el desarrollo del

\footnotetext{
7 La propuesta reconocía la autonomía de las universidades nacionales y proponía libre adhesión a un sistema cooperación interinstitucional para organizar y fortalecer el posgrado, ligado a planes de investigación y en sintonía con las necesidades nacionales y regionales en el Decreto № 1967/85 (Ministerio de Educación y Justicia, 1986).
} 
nivel, por lo que en buena medida se sustenta a través del autofinanciamiento, mediante el cobro de aranceles y matrículas en las carreras, incluso en las universidades públicas con una fuerte tradición de gratuidad en el nivel de grado.

\subsection{Características actuales de la oferta y expansión}

Ambos países convergen en la expansión cuantitativa de la oferta de carreras doctorales en la última década. En Argentina, entre 2008 y 2018, se produjo un incremento del 96\% (de 246 a 482 doctorados) y en Brasil del 76,6\% (1 284 a 2268 doctorados) $)^{8}$. A su vez, en los dos casos, las universidades públicas, especialmente las nacionales, son los espacios privilegiados que nuclean la mayor cantidad de esas carreras y en los que la oferta experimenta la mayor expansión. A continuación, la Tabla 1 muestra la distribución de doctorados por sector de gestión, en cada país en 2018.

Tabla 1

Distribución absoluta y porcentual de carreras de doctorado acreditadas, por sector público y privado en Argentina y Brasil, en 2018

\begin{tabular}{lllll}
\hline Doctorados & Argentina & $\%$ & Brasil & $\%$ \\
\hline Público & 392 & 81,3 & 1915 & 84,5 \\
Privado & 90 & 18,7 & 353 & 15,5 \\
\hline Total & $\mathbf{4 8 2}$ & $\mathbf{1 0 0}$ & $\mathbf{2 2 6 8}$ & $\mathbf{1 0 0}$
\end{tabular}

Fuente: elaboración propia en base a datos de CONEAU, 2018 y del Sistema GEOCAPES, 2019.

Sin embargo, el modo en que ese crecimiento se produce presenta diferencias. En el caso brasileño, donde el diploma de maestría es requisito para ingresar al doctorado ${ }^{9}$, las instituciones tienden a estructurar la oferta a partir de la creación y acreditación de una maestría para posteriormente avanzar con el nivel de doctorado, así en la mayoría de los casos se institucionaliza de manera predominante un programa que integra ambos niveles (PM+D). Mientras que en el caso argentino, el título exigido para acceder al doctorado es el de grado universitario o formación y méritos equivalentes pero a diferencia de la tendencia brasileña, y de manera extendida, el diploma de maestría no constituye un requisito. Esto hace que la oferta de carreras doctorales no se genere ni se articule a la creación de una maestría (PM/PD).

\footnotetext{
8 Dato de elaboración propia en base a los doctorados acreditados en CONEAU (2018), Lvovich (2010) y Sistema Geocapes (2019).

9 Aunque el Parecer de 1965 no establece la maestría como requisito para el doctorado, posteriormente esa exigencia es incorporada (Balbachevsky, 2005).
} 
Por otra parte, al incluir el análisis de las categorías de acreditación, como muestra la Tabla 2, se observa que en Brasil el sistema se estructura sobre la base de un amplio conjunto de carreras evaluadas en las categorías intermedias (5/4), 74,6\% (1.692); un menor grupo de ofertas en las categorías máximas (6/7), 18\% (407) y un número reducido en la categoría mínima de evaluación (3), 3,7\% (84). Al incorporar la clave de la distribución regional se evidencia la concentración del 51,6\% (1.171) del total de carreras en la región sureste, seguida por la región sur, 21,3\% (484) y si se consideran sólo las evaluadas como de alta calidad (6/7), la región sudeste nuclea el 65,6\% (323) de las mismas.

Tabla 2

Distribución absoluta y porcentual de los doctorados por región según categorías, Brasil, 2018

\begin{tabular}{llllllll}
\hline Región & $\mathbf{7}$ & $\mathbf{6}$ & $\mathbf{5}$ & $\mathbf{4}$ & $\mathbf{3}$ & Total & $\mathbf{\%}$ \\
\hline Sureste & 139 & 184 & 368 & 440 & 40 & 1171 & 51,6 \\
Sur & 31 & 76 & 178 & 191 & 8 & 484 & 21,3 \\
Nordeste & 9 & 28 & 106 & 196 & 23 & 362 & 16 \\
Centro-Oeste & 6 & 14 & 45 & 95 & 7 & 167 & 7,4 \\
Norte & 0 & 5 & 19 & 54 & 6 & 84 & 3,7 \\
\hline Total & $\mathbf{1 8 5}$ & $\mathbf{3 0 7}$ & $\mathbf{7 1 6}$ & $\mathbf{9 7 6}$ & $\mathbf{8 4}$ & $\mathbf{2 2 6 8}$ & $\mathbf{1 0 0}$
\end{tabular}

Fuente: elaboración propia en base a datos del Sistema GEOCAPES, 2019.

A diferencia de Brasil, como indica a continuación la Tabla 3, en Argentina casi la mitad de las carreras de doctorado alcanzan las mayores calificaciones ( $A \circ B$ ) aunque a diferencia de aquel país, la categorización no es vinculante con la obtención de un mayor financiamiento, más bien el prestigio del programa posibilita la radicación de quienes poseen una beca de formación en esos espacios. Por otra parte, sólo 38 programas $(8 \%)$ reciben la categoría más baja (C), en una proporción reducida, 69 no solicitan evaluación (14,3\%), mientras que 133 (el $27,5 \%$ ) son carreras nuevas a las que no les corresponde categorizarse.

Tabla 3

Distribución absoluta y porcentual de categorías de evaluación de carreras doctorales por región según categorías, Argentina, 2018.

\begin{tabular}{lllllll}
\hline Región & \multicolumn{3}{c}{ Categorización } & No solicitó & Total & \% \\
& A & B & C & & & \\
\hline Metropolitana & 35 & 20 & 8 & 45 & 108 & 31 \\
Centro & 48 & 24 & 11 & 8 & 91 & 26,1 \\
Bonaerense & 42 & 18 & 5 & 5 & 70 & 20,1 \\
Nuevo Cuyo & 15 & 10 & 3 & 7 & 35 & 10 \\
Noroeste & 10 & 7 & 5 & 4 & 26 & 7,4 \\
Noreste & 5 & 3 & 4 & - & 12 & 3,4 \\
Sur & 1 & 4 & 2 & - & 7 & 2,0 \\
\hline Total & $\mathbf{1 5 6}$ & $\mathbf{8 6}$ & $\mathbf{3 8}$ & $\mathbf{6 9}$ & $\mathbf{3 4 9}^{*}$ & $\mathbf{1 0 0}$
\end{tabular}

*No incluye las carreras nuevas que aún no fueron sometidas a evaluación/acreditación.

Fuente: Elaboración propia en base a datos CONEAU, 2018. 
En Argentina, casi el 80\% de las carreras se nuclea en tres regiones del país: centro, bonaerense y metropolitana, donde tradicionalmente se localizan los principales recursos e instituciones científico-universitarias.

A este panorama de la oferta se agrega, en el caso brasileño, la incorporación de la modalidad profesional a la oferta de maestría, tendencia que también se registra en México a partir del reconocimiento de los Doctorados Profesionales (DP) en el Programa Nacional de Posgrados de Calidad (PNPC), del Consejo Nacional de Ciencia y Tecnología (CONACyT) (Patiño, 2017). Su creación se vincula a la formación de estudiantes con un perfil distinto del académico y a la búsqueda de una aproximación entre el posgrado y ámbitos no universitarios de actuación profesional en los sectores público y privado (CAPES, 2014). Así, las maestrías profesionales experimentan un fuerte crecimiento en los últimos años y ya se registran avances en la creación de doctorados profesionales, orientación que en Argentina sólo es contemplada en el nivel de maestría y acotado a algunas áreas de conocimiento.

\subsection{Matrícula, doctores graduados e inserción en el sistema}

La matriz pública del sistema universitario argentino se caracteriza por el acceso sin restricciones y la gratuidad en el nivel de grado. Está conformada por 61 instituciones universitarias de gestión estatal, de las cuales 57 son universidades nacionales y 4 institutos universitarios, que concentran aproximadamente el $80 \%$ del total de la matrícula de dicho nivel y, en 2017, aglutina el 68\% de los egresados (SPU, 2019). Es en ese ámbito donde se concentran la mayoría de los programas doctorales (SPU, 2019). En cambio, el sector privado está conformado por un número mayor de universidades (63) pero con el $20 \%$ del total de la matrícula del grado y en 2017 las personas egresadas de ese sector representan $32 \%$ del total (SPU, 2019).

En contraste, en Brasil, las personas que realizan un doctorado se insertan en un sistema universitario estratificado, conformado por instituciones con modelos diferentes, objetivos múltiples y diferentes dimensiones (Dias Sobrinho y Brito, 2008). En 2018 lo integran 107 universidades públicas y 92 privadas; 13 centros universitarios públicos y 217 privados; 139 facultades públicas y 1929 privadas; 40 Institutos Federales y Centros Federales de Educación Tecnológica (Instituto Nacional de Estudos e Pesquisas Educacionais Anísio Teixeira [INEP], 2018). En ese mismo año el 75,4\% de la matrícula de grado se concentra en el sector privado y $24,6 \%$ en el sector público (INEP, 2018). 
Con una tradición de acceso regulado por procesos selectivos altamente competitivos, especialmente en las universidades públicas, el sistema universitario brasileño experimenta transformaciones a partir de la segunda mitad de la década de 2000. Así, se desarrolla un conjunto de políticas que expanden y amplían el acceso a través de programas impulsados por los gobiernos nacionales de esa época ${ }^{10}$ y adoptados por las universidades públicas, junto a un amplio crecimiento del sector privado y de la oferta de carreras universitarias a distancia. En el período 2008-2018 la matrícula del nivel de grado crece un 44,6\%, siendo ese incremento del $49,8 \%$ en el sector privado y de $33,8 \%$ en el público; mientras que en la modalidad a distancia aumenta un 182,5\% y en la presencial sólo alcanza el 25,9\% (INEP, 2018).

En esos contextos universitarios, el crecimiento de la oferta de carreras doctorales está acompañada por marcados aumentos de matrícula en ambos países, como indica la Tabla 4.

\section{Tabla 4}

Número de matrícula e incremento de las carreras doctorales por año según país, ArgentinaBrasil, 2007-2017

\begin{tabular}{llccc}
\hline Año & \multicolumn{2}{c}{ Argentina } & \multicolumn{2}{c}{ Brasil } \\
& Matrícula & Tasa de variación (\%) & Matrícula & Tasa de variación (\%) \\
\hline 2007 & 11410 & - & 49667 & - \\
2012 & 22787 & 99,7 & 79478 & 59,8 \\
2017 & 26098 & 14,5 & 112004 & 40,9 \\
\hline
\end{tabular}

Fuente: elaboración propia con base a datos de la Secretaría de Políticas Universitarias, Departamento de Información Universitaria, 2019 y del Sistema Geocapes, 2019.

En el caso de Brasil se visualiza un incremento casi del $60 \%$ de la matrícula en el período 2007-2012, que vuelve a aumentar un $40 \%$ en los cinco años siguientes. En los doctorados, en 2018, el $87,8 \%$ (100.935) del total de matrículas corresponde al sector público (GEOCAPES, 2018). En Argentina, el crecimiento es aún mayor, casi del 100\% entre 2007 y 2012, para ascender luego sólo un $14,5 \%$ entre 2012 y 2017 . Por otra parte, en 2017 , el $89 \%$ de la matrícula de carreras de doctorado se concentra en el sector público; mientras que el $56 \%$ son mujeres y el $44 \%$ varones (SPU, 2019) ${ }^{11}$. Sin embargo, la población que cursa una carrera de doctorado en 2017 es sólo un 5\% de la matrícula de nuevas personas inscriptas en ese mismo año en el nivel de pregrado y grado. En Brasil esa distancia es aún más acentuada, en 2018 representa el $1,36 \%$.

\footnotetext{
10 Principalmente del Partido de los Trabajadores (PT), durante las presidencias de Luiz Inácio Lula da Silva y de Dilma Rousseff.

${ }_{11}$ Para el caso brasileño no se incluye el dato sobre la distribución de la matrícula entre hombres y mujeres por no estar incluido en las estadísticas del sistema GEOCAPES.
} 
Ambos países muestran un importante desequilibrio regional en la distribución de matrículas y de graduados, con una fuerte concentración en las zonas que tradicionalmente nuclean los mayores recursos e instituciones científico-universitarias.

En Argentina el $82,4 \%$ de la matrícula de los programas de doctorado y el $84,6 \%$ de los graduados se nuclea en las zonas metropolitana, bonaerense y centro, como indica la Tabla 5.

Tabla 5

Distribución absoluta y porcentual de estudiantes y graduados en programas doctorales por región, Argentina, 2017

\begin{tabular}{lllll}
\hline CPRES* & Matrícula & $\%$ & Egresados & $\%$ \\
\hline Metropolitano & 7558 & 29 & 802 & 38,1 \\
Bonaerense & 7510 & 28,8 & 500 & 23,7 \\
Centro & 6409 & 24,6 & 480 & 22,8 \\
Cuyo & 1638 & 6,2 & 131 & 6,2 \\
Noroeste & 1610 & 6,2 & 92 & 4,4 \\
Noreste & 864 & 3,3 & 64 & 3 \\
Sur & 509 & 1,9 & 37 & 1,8 \\
\hline Total & $\mathbf{2 6} \mathbf{0 9 8}$ & $\mathbf{1 0 0}$ & $\mathbf{2 1 0 6}$ & $\mathbf{1 0 0}$ \\
\hline
\end{tabular}

*CPRES: Consejos Regionales de Planificación de la Educación Superior

Fuente: elaboración propia con base a datos SPU, 2019.

La disparidad regional es aún mayor en el Brasil y aunque hubo un crecimiento general de estas carreras en las últimas décadas sólo dos regiones, sur y sureste, concentran más del $70 \%$ de la matrícula y los graduados, mientras que en las regiones norte y centro-oeste, ese porcentaje es inferior al 10\%, como muestra la Tabla 6.

Tabla 6

Distribución absoluta y porcentual de estudiantes y graduados de carreras doctorales por región en Brasil, 2017

\begin{tabular}{lllll}
\hline Región & Matrícula & $\%$ & Doctores & $\%$ \\
\hline Sureste & 59824 & 52,2 & 12273 & 56,8 \\
Sur & 22610 & 19,8 & 4339 & 20 \\
Nordeste & 21107 & 18,4 & 3284 & 15,2 \\
Centro-Oeste & 7242 & 6,3 & 1205 & 5,6 \\
Norte & 3732 & 3,3 & 526 & 2,4 \\
\hline Total & $\mathbf{1 1 4 5 1 5}$ & $\mathbf{1 0 0}$ & $\mathbf{2 1 5 9 1}$ & $\mathbf{1 0 0}$
\end{tabular}

Fuente: elaboración propia con base a datos del Conicet, 2019 y del Sistema Geocapes, 2019

En ese país también se verifica la incorporación de un importante número de personas con título de doctorado al sistema de enseñanza del posgrado, en el marco del proceso de expansión de la oferta de carreras. En 2008 se registran 41995 personas con cargo 
permanente en el profesorado con título de doctorado en ese espacio, mientras que diez años después ascienden a 82028.

Las personas con título de doctorado en el cuerpo del profesorado de las universidades de Argentina representan sólo el 10,8\% del total de la población (SPU, 2015), cuestión que resulta, en parte, de la desarticulación entre las políticas científicas de formación recursos calificados y las académico-universitarias, como también de la heterogeneidad de criterios de evaluación existentes en los concursos públicos y abiertos de antecedentes y oposición para acceder a los cargos y a la carrera docente en las universidades. Respecto a la inserción profesional de las personas con título de doctorado, otros estudios problematizan, además, la formación predominantemente académica de los programas y más recientemente, el estrangulamiento laboral, que dificulta su ingreso a los ámbitos científicos y universitarios existentes (Unzué y Emiliozzi, 2017). En Brasil, la modalidad profesional, ya instalada en el nivel de maestría e incipiente en el doctorado, constituye una acción generada por el propio sistema para ampliar y diversificar la inserción de las personas graduadas.

\subsection{Fomento estatal a la formación doctoral}

Otro aspecto divergente en ambos casos nacionales es la articulación entre el sistema de posgrado y el científico tecnológico en la forma de acceso a becas doctorales y el ingreso a programas. En el país austral existen distintos dispositivos de becas y, como se dijo anteriormente, el más extendido es el de las de formación doctoral otorgadas por el CONICET. Las becas se asignan directamente al estudiantado seleccionado, quien - una vez asignada la beca- tiene un plazo de seis meses para formalizar su inscripción a un programa de doctorado acreditado por la CONEAU. En cambio, en Brasil, los destinatarios de las becas de formación para maestría y doctorado, con dos y cuatro años de duración respectivamente, provenientes de la CAPES y el CNPq, son los programas de posgrado stricto sensu, quienes están encargados de la selección de las personas que se postulan, en función de las plazas disponibles. La distribución entre los programas se encuentra principalmente articulada a la categorización atribuida por el sistema de evaluación CAPES, aunque a partir de 2019 comienza a discutirse la incorporación de otros criterios. También existen becas posdoctorales orientadas a la radicación de jóvenes con título de doctorado y a la internacionalización, que ocupa un lugar destacado en el PNPG y en el sistema de evaluación de carreras.

En Argentina, a lo largo de los años, la denominación de las becas en los reglamentos del CONICET ha ido mutando en dos sentidos: el primero, muestra un mayor acercamiento al 
nivel de posgrado (en especial, al título de doctorado y posdoctorado) en detrimento de anteriores denominaciones vinculadas a los distintos peldaños en la formación en investigación (por ejemplo, la actividad de iniciación y perfeccionamiento). En segundo lugar, se observa un desplazamiento paulatino de la formación de maestría en pos del fortalecimiento de la beca doctoral, en tanto titulación mínima requerida para el ingreso a la carrera científica. A partir de 2014 se definen tres categorías centrales de becas: doctorales, para dicha formación y con una duración de cinco años; de finalización de doctorado, para completar esos estudios y posdoctorales, estas dos últimas otorgadas por período de dos años ${ }^{12}$.

En ambos países los datos muestran que la expansión de los doctorados se asocia al incremento de la inversión pública en dichas becas durante el período 2000-2015 en el caso brasileño y entre 2005 y 2015 en el argentino, tendencia que se verifica en la Tabla 7.

Tabla 7

Número de becas e incremento de la formación doctoral por año, según país, Argentina- Brasil, 2007-2018

\begin{tabular}{ccccc}
\hline Año & \multicolumn{2}{c}{ Argentina } & \multicolumn{2}{c}{ Brasil } \\
& Becas $^{*}$ & Tasa de variación (\%) & Becas & \\
\hline 2007 & 5844 & - & 11191 & - \\
2010 & 6302 & 7,8 & 21941 & 96 \\
2015 & 7815 & 28,5 & 42779 & 94,9 \\
2016 & 7814 & 0 & 43188 & $-0,9$ \\
2017 & 7914 & 0 & 44316 & 2,6 \\
2018 & 7533 & $-4,8$ & 44530 & 0,4 \\
\hline
\end{tabular}

${ }^{*}$ El dato incluye las becas de finalización de doctorado, otorgadas por el CONICET en Argentina a partir de 2014.

Fuente: elaboración propia en base a datos del CONICET, 2019 y del Sistema Geocapes, 2019.

En el primer país, existe una variación incremental del $96 \%$ y del $94,6 \%$ en las becas doctorales entre 2007 y 2010 y entre 2010 y 2015 respectivamente, mientras que como se desprende de la Tabla 8, en las posdoctorales resulta exponencial al alcanzar un aumento del 472\% en 2010, respecto de 2007 y un 174\% en 2015, en relación con los cinco años anteriores. Por su parte, en Argentina, la política de formación de recursos altamente calificados, a través de becas CONICET, comienza a implementarse recién a partir del 2003, por lo que las becas doctorales aumentan un 416,4\% en 2005 respecto del 2000. En la Tabla 7, se observa un incremento $7,8 \%$ en 2010 respecto de 2007 y del $28 \%$ en 2015, en relación con los cinco años

\footnotetext{
12 Aunque en menor magnitud, existen becas co-financiadas con otros organismos científicos y países latinoamericanos, destinadas a unidades ejecutoras y centros de investigaciones y transferencia (CIT) del CONICET, entre otras.
} 
anteriores; mientras que en la Tabla 8, el aumento en las posdoctorales es del 105,3\% y del $57 \%$ en esos mismos períodos. En un escenario financiero de ajuste y de incertidumbre para las políticas del sector, a partir de 2016 y en los siguientes dos años, en el Brasil se visualiza un estancamiento en el número de becas doctorales, aún mayor y de disminución en las posdoctorales. En Argentina, ocurre la misma tendencia en ambos tipos de becas entre 2016 y 2017 mientras que se profundiza el recorte en las doctorales y no hay crecimiento en las posdoctorales hacia 2018.

\section{Tabla 8}

Número de becas e incremento de la formación posdoctoral por año, según país, ArgentinaBrasil, 2007-2018

\begin{tabular}{|c|c|c|c|c|}
\hline \multirow[t]{2}{*}{ Año } & \multicolumn{2}{|c|}{ Argentina } & \multicolumn{2}{|c|}{ Brasil } \\
\hline & Becas & Tasa de variación (\%) & Becas & Tasa de variación (\%) \\
\hline 2007 & 779 & - & 479 & - \\
\hline 2010 & 1600 & 105,3 & 2734 & 472 \\
\hline 2015 & 2512 & 57 & 7486 & 174 \\
\hline 2016 & 2235 & -1 & 6999 & -7 \\
\hline 2017 & 2038 & $-8,8$ & 7168 & 2 \\
\hline 2018 & 2097 & 2,8 & 7075 & -1 \\
\hline
\end{tabular}

Fuente: elaboración propia en base a datos del CONICET, 2019 y del Sistema Geocapes, 2019.

Una novedad introducida por el CONICET es la incorporación de una línea de orientación en el sistema de becas doctorales y posdoctorales hacia temas estratégicos, vinculada al Plan Nacional de Ciencia, Tecnología e Innovación (MINCyT, 2012), que define por primera vez en el país seis grandes temáticas prioritarias para el desarrollo de la I+D (Agroindustria, Ambiente y Desarrollo Sustentable, Desarrollo Social, Energía, Industria y Salud) y que entre los ejes transversales que atraviesan a las políticas de ciencia, tecnología e innovación, reconoce al género y a la equidad de género en tanto estrategia de desarrollo institucional. Desde 2012 esos temas son adoptados como referencia en el Programa de Becas Doctorales y Posdoctorales y en la asignación de vacantes a la Carrera de Investigador Científico del CONICET, en paralelo a la tradicional convocatoria en temas abiertos por grandes áreas disciplinares. En esos años, los temas estratégicos alcanzan mayor preponderancia en la asignación de becas doctorales y posdoctorales del organismo al representar el $16,7 \%$ del total de becas asignadas en 2012 y el 25,2\% en 2017; mientras que desde 2016 constituyen la primera prioridad (ante otras como las de distribución geográfica o las de fortalecimiento institucional) en la asignación de vacantes (Jeppensen et al., 2018; Rovelli, 2017). No obstante, 
aún resulta incipiente la articulación entre las becas orientadas a temas estratégicos y una oferta de formación doctoral definida, o bien con agendas de investigación que se correspondan con esos lineamientos en las universidades donde se radican los programas.

En el caso de Brasil, la orientación de recursos humanos calificados en áreas estratégicas ocurre más tempranamente que en Argentina. Los PNPG 2005-2010 y 2011-2020 destacan la importancia de promover la formación de recursos humanos en dichas áreas, definidas por temas prioritarios: Amazonia y el Mar; Biotecnología; Fármacos, Medicamentos y Vacunas; Materiales Avanzados; Nanotecnología, Microelectrónica; Espacio, Defensa y Energía Nuclear vinculados a las áreas de Biotecnología, Ciencias Biológicas, Ingenierías, Ciencias de la Computación, Física, Química, Ciencias de la Salud, Ciencias Ambientales y Biodiversidad (CAPES, 2018a). Si bien esa agenda ocupa un espacio relevante en acciones de fomento a la investigación, no interfiere en la distribución de becas doctorales que, como ya fue apuntado, se rige por otros criterios.

\section{Conclusiones}

Con una configuración histórica más temprana, el posgrado stricto sensu brasileño se institucionaliza importando el modelo norteamericano y se articula a la organización del sistema departamental establecido a fines de la década de 1960 en todas las universidades públicas. En la década siguiente se crea el sistema de evaluación por pares para esas carreras que, con modificaciones, continúa hasta la actualidad. De esta manera, se destaca el gobierno y la administración centralizada de la CAPES, donde el financiamiento para el posgrado queda sujeto a la acreditación y categorización periódica.

En el caso argentino, si bien surge una oferta de doctorado incipiente a principios del siglo XX, se trata, en muchos casos, de proyectos inconclusos como resultado de los vaivenes políticos e institucionales, los que no logran cristalizarse en una propuesta más extendida de educación de posgrado. Recién a partir de la década de 1990 y como resultado del influjo de distintas tradiciones internacionales (entre las que predomina el modelo francés de corte más profesional) logra conformarse una oferta estable de evaluación y acreditación de las carreras de posgrado. Esa configuración se caracteriza por la centralización de los procesos de evaluación/acreditación en la CONEAU, la escasa vinculación con el financiamiento de las carreras y por un entramado institucional donde las universidades adquieren una mayor autonomía. A su vez, entre esa agencia de evaluación, los programas doctorales radicados en las universidades y los organismos de ciencia y tecnología pervive una planificación y 
coordinación débil, en particular en relación con aquellas políticas orientadas hacia la promoción de la formación doctoral.

En el período estudiado, y aunque en diferentes escalas para cada caso nacional, se identifica un marcado aumento de carreras doctorales. En Brasil, la tendencia se expresa en la expansión de programas de maestría y doctorado (modelo PM+PD) y en una reciente diversificación de la modalidad académica y profesional. Esta última se expande como un instrumento para la formación de personas graduadas con un perfil diferenciado del académico y para responder a demandas del mercado profesional. En Argentina, el crecimiento de los doctorados se explica por la incidencia indirecta de las becas de formación financiadas por el CONICET y en menor medida, por otras instituciones, así como por la ausencia de una tradición de la maestría como requisito para acceder al doctorado (modelo PD).

En ambos países, tanto el aumento de la matrícula como de personas graduadas con título de doctorado ocurre de manera preponderante en el sector público, en dos sistemas universitarios que se diferencian por el nivel de privatización del nivel de grado y que continúan evidenciando una alta inequidad regional en la distribución de la oferta y los recursos calificados. A su vez, la expansión del propio sistema de posgrado brasileño, a diferencia del caso argentino, ha incorporado un importante número de personas graduadas con título de doctorado.

En ambos casos la expansión es acompañada por una importante inversión en la política de becas, organizada a través de distintas articulaciones entre el sistema universitario y el científico tecnológico. A lo anterior se suman instrumentos innovadores en la orientación de la formación hacia temas estratégicos, en el caso argentino y reconfiguraciones de la oferta de carreras orientadas a la inserción profesional de graduados en espacios no académicos, en el caso brasileño. También se reconocen desafíos pendientes en torno al egreso y la inserción de las personas graduadas, que aún requieren de respuestas más integrales en el plano de las políticas, de la coordinación de los sistemas y de su vinculación con los entornos sociales y productivos. Finalmente, ambos países presentan en los últimos años indicadores de retracción y desinversión que plantean incertidumbres en relación con la continuidad de esa tendencia creciente. Si bien en Argentina la asunción de una nueva gestión gubernamental, en diciembre de 2019, ha logrado recomponer el papel estratégico de las políticas de formación doctoral y de manera más amplia, de la ciencia y la tecnología para el desarrollo del país, la inversión en la formación doctoral deberá seguir siendo analizada en futuros estudios. 
Por último, en el marco de un escenario social y educativo reconfigurado a partir de la pandemia Covid-19, los programas y la formación doctoral enfrentan nuevos desafíos. Entre otros, existe la necesidad de crear y/o ampliar tecnologías de información y comunicación que faciliten la interacción, formación, supervisión y graduación del estudiantado; la posibilidad de garantizar la continuidad de un financiamiento sostenido y adecuado, así como también la promoción de nuevas formas de colaboración internacional y el fortalecimiento de los de procesos de apertura en el acceso a datos de investigación y a publicaciones científicas. Estos hechos le otorgan un renovado interés a nuestro estudio, pues seguramente inaugurarán procesos novedosos en relación con la estructura, regulación y dinámica de la oferta, matrícula y graduación en el espacio social del posgrado a nivel regional e internacional, a los que será preciso atender en próximas investigaciones.

\section{Referencias}

Araujo, Sonia M., Balduzzi, Matilde M., Corrado, Rosana. y Walker, Verónica. (2016). Evaluación y acreditación de los posgrados en Argentina, Brasil y Paraguay. Aproximaciones comparativas. En Nora Z. Lamfri (Coord.), Los posgrados en Argentina, Brasil y Paraguay: aproximaciones comparadas en contextos de evaluación de la calidad de la educación superior (pp. 147-194). Córdoba: Encuentro Grupo Editor. Recuperado de

http://nemercosur.siu.edu.ar/webnucleo/documentos/LOS POSGRADOS EN ARGEN TINA BRASIL Y PARAGUAY.pdf

Balbachevsky, Elizabeth. (2005). A pós-graduação no Brasil: novos desafios para uma política bem-sucedidas. En Colin Brock e Simón Schwartzman. Os desafios da educação no Brasil (pp. 275-304). Rio de Janeiro: Nova Fronteira. Recuperado de https://files.cercomp.ufg.br/weby/up/67/o/Pos-Graduacao Brasil 2.pdf

Barsky, Osvaldo. (1995). El sistema de posgrado en la Argentina (Serie Estudios y Propuestas). Buenos Aires: MECyT.

Bekerman, Fabiana. (2019). La investigación científica argentina en dictadura. Transferencias y desplazamientos de recursos (1974-1983). Mendoza, Argentina: EDIUNC.

Bray, Mark., Adamson, Bob. y Mason, Mark. (2010). Educación Comparada. Enfoques y Métodos. Buenos Aires: Granica.

Calderon, Adolfo-Ignacio., da Silva Wargas, Bruna M., Borochovicius, Eli., Wandecil, Marco., Piason Breglio Pontes, Marina., Marinho de Barros, Adelir A., Brázier, Fabio. y Batista de Sousa Koide, Adriana. (2019). Doutorado Profissional em Educação: tendências em universidades de classe mundial contextualizadas nos rankings acadêmicos internacionai. Revista Praxis Educativa, 14(1), 138-162. Recuperado de https://revistas2.uepg.br/index.php/praxiseducativa/article/view/12007/209209210551 
Castro, Cláudio de Moura. (2005). A hora do mestrado profissional. Revista Brasileira de PósGraduação, 2(4), 16-23. $\quad$ Recuperado de http://ojs.rbpg.capes.gov.br/index.php/rbpg/article/view/73/70

Cirani, Claudia B. S., Campanario, Milton A., Silva, Heloisa. H. M. (2015). A evolução do ensino da pós-graduação senso estrito no Brasil: análise exploratória e proposições para pesquisa. Avaliação, 20(1), 163-187. Recuperado de https://www.scielo.br/scielo.php?pid=S1414-

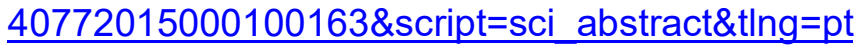

Comisión Nacional de Evaluación y Acreditación Universitaria (CONEAU). (2018). Posgrados acreditados de la República Argentina. Ciudad Autónoma de Buenos Aires: CONEAU. Recuperado https://www.coneau.gob.ar/archivos/publicaciones/CatalogoPosgrados 2019.pdf

Consejo Nacional de Investigaciones Científicas y Técnicas (CONICET). (2019). Resultados Convocatoria Becas Doctorales y Posdoctorales. Recuperado de https://convocatorias.conicet.gov.ar/resultados-becas-2018/

Conselho Federal de Educação da República Federativa do Brasil. (2005). Parecer n 977/65, aprobado el 3 de diciembre de 1965. Revista Brasileira de Educação, (30), 162-173. Recuperado de https://www.scielo.br/pdf/rbedu/n30/a14n30.pdf

Coordenação de aperfeiçoamento de pessoal de nível superior (CAPES). (2010). Plano Nacional de Pós-graduação 2011-2020. Ministério da Educação. Recuperado de https://www.capes.gov.br/plano-nacional-de-pos-graduacao

Coordenação de aperfeiçoamento de pessoal de nível superior (CAPES). (2014). Egressos da Pós-graduação: Áreas estratégicas. Relatório Técnico da DAV. Ministério da Educação. Recuperado https://www.capes.gov.br/images/stories/download/avaliacao/19122018 Cartilha-DAVEgressos.pdf

Coordenação de aperfeiçoamento de pessoal de nível superior (CAPES). (2018a). Educação doutoral: reformas e tendências. Brasil: Ministério da Educação. Recuperado de http://www.prppg.ufpr.br/site/wp-content/uploads/2020/07/19122018 cartilha-daveducacao.pdf

Coordenação de aperfeiçoamento de pessoal de nível superior (CAPES). (2018b). Proposta de Aprimoramento do Modelo de Avaliação da PG Documento Final da Comissão Nacional de Acompanhamento do PNPG 2011-2020. Ministério da Educação. Recuperado de https://www.capes.gov.br/images/novo portal/documentos/PNPG/2018 PNPG CS Av aliacao Final $10 \quad 10 \quad 18$ CS FINAL $17 \quad 55 . p d f$

Coordenação de aperfeiçoamento de pessoal de nível superior (CAPES). (2020). Relatório 2019. Proposta de Aprimoramento da Avaliação da Pós-graduação Brasileira para o Quadriênio 2021-2024 - Modelo Multidimensional. Recuperado de https://www.gov.br/capes/pt-br/centrais-de-conteudo/25052020-relatorio-final-2019comissao-pnpg-pdf 
Decreto Institui a Política de Dados Abertos do Poder Executivo federal, $N^{\circ} 8777$. (2016). Recuperado de http://www.planalto.gov.br/ccivil 03/ Ato20152018/2016/Decreto/D8777.htm

De La Fare, Mónica., y Lenz, Sylvia. (2012). El Posgrado en el Campo Universitario: expansión de carreras y productividad de tesis en la Argentina. Buenos Aires: IEC-CONADU, Universidad Nacional de General Sarmiento.

Dias Sobrinho, José. y de Brito, Márcia R. F. (2008). La educación superior en Brasil: principales tendencias y desafíos. Revista de Avaliação da Educação Superior, 13(2), 487-507. Recuperado de https://www.scielo.br/scielo.php?script=sci arttext\&pid=S1414$40772008000200011 \&$ lng=en\&nrm=iso\&tlng=es

Emiliozzi, Sergio. (2013). Políticas para la formación de recursos humanos calificados em argentina y Brasil. En Martín Unzué y Sergio Emiliozzi (Comp.), Universidad y políticas públicas: ¿En busca del tiempo perdido? Argentina y Brasil en perspectiva comparada (pp. 9-48). Buenos Aires: Imago Mundi.

Gallardo, Osvaldo. (2015). Trayectorias de formación de investigadores del CONICET. Revista Sociedad, (34), 129-148. Recuperado de http://www.sociales.uba.ar/wpcontent/blogs.dir/219/files/2016/03/23-MARZO-REVISTA-SOCIALES.pdf

García de Fanelli, Ana M. (1996). Estudios de posgrado en la Argentina: Alcances y limitaciones de su expansión en las universidades públicas (Serie Educación Superior). Buenos Aires: CEDES.

García, Lucía B. (Coord.) (2016). Situación actual del posgrado en Argentina, Brasil y Paraguay: carreras, estudiantes y egresados. En Nora Z. Lamfri (Coord.). Los posgrados en Argentina, Brasil y Paraguay: aproximaciones comparadas en contextos de evaluación de la calidad de la educación superior (pp. 147-194). Córdoba: Encuentro Grupo Editor. Recuperado de http://nemercosur.siu.edu.ar/webnucleo/documentos/LOS POSGRADOS EN ARGEN TINA BRASIL Y PARAGUAY.pdf

GEOCAPES. (2019). Sistema de Informações Georreferenciadas. Recuperado de https://geocapes.capes.gov.br/geocapes/

Hortale, Virginia Alonso., Moreira, Carlos Otávio Fiúza., Bochner, Rosany, y Leal, Maria do Carmo. (2014). Trajetória profissional de egressos de cursos de doutorado nas áreas da saúde e biociências. Revista de Saúde Pública, 48(1), 1-9. Recuperado de https://doi.org/10.1590/S0034-8910.2014048004629

Hurtado, Diego. (2015). La cultura científico-tecnológica argentina en contexto democrático: tres etapas. En Sebastián Mauro, Damián Del Valle y Federico Montero (Comps.), Universidad pública y desarrollo: innovación, inclusión y democratización del conocimiento (pp. 34-47). Buenos Aires: IEC - CONADU; Ciudad Autónoma de Buenos Aires; CLACSO. Recuperado de http://biblioteca.clacso.edu.ar/clacso/se/20160301022159/universidad publica.pdf 
Instituto Nacional de Estudos e Pesquisas Educacionais (INEP). (2018). Censo de la Educação Superior 2018. Recuperado de: http://portal.inep.gov.br

Jeppensen, Cynthia., Bentura, Martín., Goldberg, Mariela. y Fernández López, Paula. (2018). La formación de doctorado y el programa de becas de Temas Estratégicos en CONICET. Descripción y aportes para una evaluación programática. Ponencia presentada en las $X$ Jornadas de Sociología de la UNLP. Ensenada: UNLP. Recuperado de http://sedici.unlp.edu.ar/handle/10915/82212

Jeppensen, Cynthia., Golberg, Mariela., Szpeiner, Alfonsina. y Rodríguez Gauna, María Cecilia. (2015). Estrategias, instrumentos y resultados de la política pública de RRHH en los últimos diez años. Revista Sociedad, (34), 73-84. Recuperado de http://www.sociales.uba.ar/wp-content/blogs.dir/219/files/2016/03/23-MARZO-

REVISTA-SOCIALES.pdf

Krotsch, Pedro. (1996). El postgrado en Argentina: una historia de discontinuidad y fragmentación. Pensamiento Universitario, 4, (4-5), 43-56.

Kuenzer, Acacia Zeneida., y Moraes, Maria Célia Marcondes de. (2005). Temas e tramas na pós-graduação em educação. Educação \& Sociedade, 26(93), 1341-1362. Recuperado de https://doi.org/10.1590/S0101-73302005000400015

Ley de Educación Superior (LES), $N^{\circ}$ 24.521. (1995). Recuperado de http://servicios.infoleg.gob.ar/infoleglnternet/anexos/25000-29999/25394/texact.htm

Lei Plano Nacional de Educação, No 13.005. (2014). Recuperado de http://www.planalto.gov.br/ccivil 03/ ato2011-2014/2014/lei//13005.htm

Lovisolo, Hugo. (2000). Vecinos Distantes. Universidad y Ciencia en Argentina y Brasil. Buenos Aires: Libros del Zorzal.

Lvovich, Daniel. (2010). Resultado e impactos de los programas de apoyo a la formación de posgrado en Argentina. Lucas, Luchilo (Comp.), Formación de posgrado en América Latina. Políticas de apoyo, resultados e impactos (pp. 51-86). Ciudad de Buenos Aires: Eudeba.

Mendes, Jussara M. R., Werlang, Rosangela., y Santos, Andreia M. (2017). Pós-graduação em Serviço Social no Brasil: há uma pedra no caminho. Revista Katálysis, 20(2), 175183. Recuperado de https://doi.org/10.1590/1982-02592017v20n2p175

Ministerio de Ciencia, Tecnología e Innovación Productiva (MINCyT). (2012). Plan Nacional de Ciencia, Tecnología e Innovación Argentina Innovadora 2020. Lineamientos estratégicos 2012-2015. Buenos Aires: MINCyT. Recuperado de https://www.argentina.gob.ar/sites/default/files/pai2020.pdf

Ministerio de Educación y Justicia. (1986). Decreto Sistema Interuniversitario del Cuarto Nivel (SICUN). N $N^{\circ} 1967$. En Normas que regulan el funcionamiento del Consejo Interuniversitario Nacional (C.I.N.) y el Sistema Interuniversitario de Cuarto Nivel (S.I.C.U.N.) (pp. 14-20). Buenos Aires. Recuperado de https://core.ac.uk/download/pdf/32378359.pdf 
Moritz, Gilberto O., Moritz, Mariana O., Pereira, Mauricio F., y Maccari, Emerson A. (2013). A pós-graduação brasileira: evolução e principais desafios no ambiente de cenários prospectivos. Future Studies Research Journal: Trends and Strategies, 5(2), 3-3. Recuperado de http://www.spell.org.br/documentos/ver/21108/a-pos-graduacaobrasileira--evolucao-e-principais-desafios-no-ambiente-de-cenarios-prospectivos/i/pt-br

Negret, Fernando. (2008). A identidade e a importância dos mestrados profissionais no Brasil e algumas considerações para a sua avaliação. Revista Brasileira De Pós-Graduação, 5(10), 217-225. Recuperado de http://ojs.rbpg.capes.gov.br/index.php/rbpg/article/view/152/146

Organización para la Cooperación y el Desarrollo Económico (OCDE). (2016). OECD Science, Technology and Innovation Outlook 2016. Recuperado de http://dx.doi.org/10.1787/sti in outlook-2016-en

Patiño, Josefina. (2017). Doctorados profesionales: concepciones y debate. Entrevista a Luis Ponce Ramírez. Revista Electrónica de Investigación Educativa, 19(4), 1-15. Recuperado de http://redie.uabc.mx/redie/article/view/1741

Piovani, Juan I., y Krawczyk, Nora. (2017). Los estudios comparativos: algunas notas históricas, epistemológicas y metodológicas. Educaçao \& Realidade, 42(3), 821-840. Recuperado de http://www.memoria.fahce.unlp.edu.ar/art revistas/pr.8927/pr.8927.pdf

Rovelli, Laura Inés. (2017). Expansión reciente de la política de priorización en la investigación científica de las universidades públicas en Argentina. Revista iberoamericana de educación superior, 8(22), 103-121. Recuperado de https://www.ries.universia.unam.mx/index.php/ries/article/view/231

Schwartzmann, Simon. (1991). A space for Science: the Development of the Scientific Community in Brazil. Pennsylvania: Pennsylvania State Universtiy Press. Recuperado de http://www.schwartzman.org.br/simon/space/summary.htm

Secretaría de Políticas Universitarias (SPU). (2019). Sistema de Consultas de Estadísticas Universitarias. Departamento de Información Universitaria. Recuperado de: http://estadisticasuniversitarias.me.gov.ar/\#/home/2

Secretaría de Políticas Universitarias (SPU). (2015). Anuario 2015. Estadísticas Universitarias Argentinas. Argentina: Ministerio de Educación, Presidencia de la Nación. Departamento de Información Universitaria. Recuperado de: http://informacionpresupuestaria.siu.edu.ar/DocumentosSPU/diu/Anuario\%202015\%20\%20ESTAD\%C3\%8DSTICAS\%20UNIVERSITARIAS\%20ARGENTINAS\%20$\% 20$ SPU $\% 20 . p d f$

Unzué, Martín., y Emiliozzi, Sergio. (2017). Las políticas públicas de Ciencia y Tecnología en Argentina: un balance del período 2003-2015. Temas debates, (33), 13-33. Recuperado de $\quad$ http://www.scielo.org.ar/scielo.php?script=sci arttext\&pid=S1853984X2017000100001\&lng=es\&tlng=es 
Unzué, Martín. (2011). Claroscuros del desarrollo de los posgrados en Argentina. Sociedad, Revista de la Facultad de Ciencias Sociales de la Universidad de Buenos Aires, (29/30), 127-148. Recuperado de http://www.sociales.uba.ar/wp-content/uploads/Sociedad2930-FINAL2.pdf

Unzué, Martín. (2013). Autonomía, evaluación y políticas públicas. Tendencias y límites en los sistemas universitarios de Argentina y Brasil. En Martín Unzué y Sergio Emiliozzi (Comp.), Universidad y políticas públicas: ¿En busca del tiempo perdido? Argentina y Brasil en perspectiva comparada (pp. 9-48). Buenos Aires: Imago Mundi.

Unzué, Martín. (2015). Nuevas políticas públicas de formación de doctores en Argentina. Revista Sociedad, (34), 15-38. Recuperado de http://www.sociales.uba.ar/wpcontent/blogs.dir/219/files/2016/03/23-MARZO-REVISTA-SOCIALES.pdf 
Revista indizada en

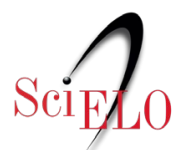
redalyc latindex

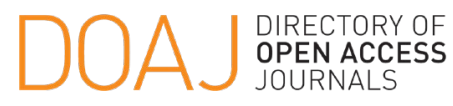

Distribuida en las bases de datos:

- Dialnet
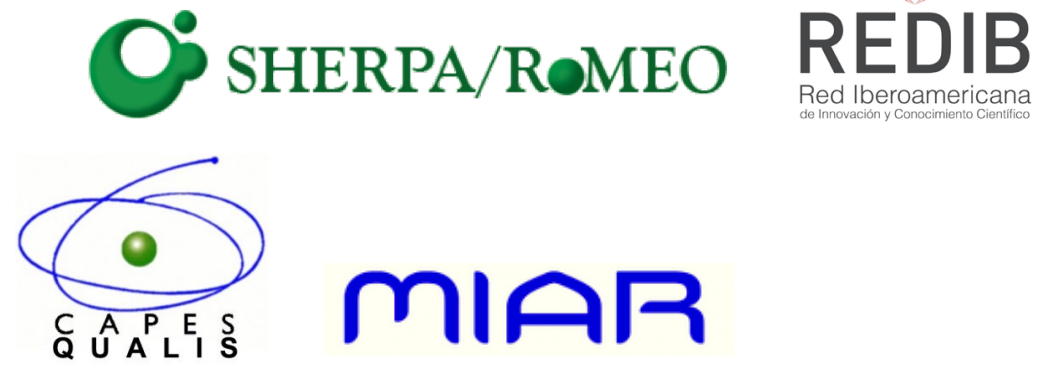

MIAR 